\title{
El APAS (Ariel Performance Analysis System) como medio de evaluación fisioterapéutica de la marcha en un paciente espástico
}

\author{
Carolina Calderón Villamizar* \\ Jorge Enrique Correa** \\ Martha Jiménez $V$.***
}

\begin{abstract}
Resumen
El propósito de la investigación fue realizar un análisis del APAS (Ariel Performance Analysis System) como instrumento de evaluación fisioterapéutica de la marcha en un paciente espástico, mejorando los procesos de evaluación, dentro de la fase de secuelas de la parálisis cerebral. Se desarrolló a través de un estudio descriptivo de caso con un paciente con parálisis cerebral $(P C)$ de género masculino de 32 años de edad, donde se demostró los beneficios en el uso del Software especializado como instrumento de medición el cual mejora la evaluación de la marcha. El análisis cinemático de la marcha con el APAS permitió describir de manera precisa, el comportamiento osteocinemático de las extremidades inferiores, durante el ciclo de la marcha en los tres planos de movimiento en el espacio. Los resultados muestran la restricción en el desplazamiento articular generados por las contracturas articulares y la asimetría muscular que lleva a una alteración del ciclo de la marcha y un aumento del consumo energético. Los datos arrojados por el APAS permiten un análisis preciso de la marcha en la cual se puede observar los distintos planos de movimiento, lo que aporta al proceso de evaluación tradicional y a la posible intervención fisioterapéutica, ya que precisa el grado de las insuficiencias y compensaciones funcionales en el movimiento.
\end{abstract}

Palabras clave: fisioterapia, evaluación, marcha espástica, cinemática.

\section{Introducción}

La magnitud creciente de los nacimientos con parálisis cerebral y la instauración de sus secuelas, sumado al aumento del deseo por la recuperación funcional de sus familiares, la presión ejercida por la población afectada y por influyentes sectores sociales, ha puesto en discusión y como primer plano de la agenda sanitaria y social, la necesidad de mejorar los procesos de rehabilitación de las personas con secuelas funcionales motoras haciendo parte esencial el profesional de
Fisioterapia en los procesos de rehabilitación y reinserción social. (Hart y Miller, 1993).

\footnotetext{
* Estudiante de Fisioterapia, Escuela Colombiana de Rehabilitación.

** Asesor temático. Fisioterapeuta. Especialista en Docencia Universitaria y Gerencia en Organizaciones de Salud. Candidato a maestría de Fisiología de la Universidad Nacional. Docente de la Escuela Colombiana de Rehabilitación y director de la línea de Investigación en Ejercicio Físico y Desarrollo Humano de la Universidad del Rosario.

**** Asesora metodológica. Psicóloga, especialista en Análisis de Datos, y Diplomada en Población y Desarrollo.
} 
La parálisis cerebral constituye un importante problema social y económico, así como un reto para el sistema de salud colombiano. Es la causa más frecuente de enfermedad neurológica infantil y su frecuencia va en aumento sobre todo en los países de Latinoamérica (Moreno-Gea y Blanco Sánchez, 2000).

En el ámbito mundial constituye la primera causa de discapacidad infantil, sobre todo en países subdesarrollados. En el caso del nuestro, tiene una tendencia ascendente, superada sólo por las enfermedades cardiovasculares y el cáncer, con una tasa cruda de mortalidad de 66,2 × 100. 000 habitantes.

Se estima por las Asociaciones Unidas de Parálisis Cerebral que más de 500.000 norteamericanos padecen parálisis cerebral. A pesar del progreso en prevenir y tratar ciertas causas de la parálisis cerebral, el número de niños y adultos afectados ha aumentado durante los últimos 30 años. En la mayoría de los casos no se encontró causa alguna. Estas conclusiones del estudio perinatal del Instituto Nacional de Trastornos Neurológicos y Apoplejía (NINDS) han alterado profundamente las teorías médicas sobre la parálisis cerebral y han motivado a los investigadores a explorar causas alternas (Anderson y Mattsson, 2001).

Al mismo tiempo, la investigación biomédica también ha conducido a cambios significativos en el conocimiento, diagnosis y tratamiento de personas con parálisis cerebral. La identificación de los bebés con parálisis cerebral en un estado temprano de la vida, les da oportunidad de desarrollar al máximo sus capacidades. La investigación biomédica ha conducido a mejores técnicas diagnósticas, tales como imagen cerebral avanzada y el análisis moderno funcional de patrones motores como la marcha espástica, entre otras. Ciertas condiciones conocidas como causantes de la parálisis cerebral, tales como rubéola e ictericia, actualmente pueden ser prevenidas o tratadas. En este sentido la Fisioterapia, la Terapia
Ocupacional y la Fonoaudiología también desarrollan destrezas del movimiento y del habla promoviendo el desarrollo social y emocional de las personas con parálisis cerebral.

En la actualidad, los procesos de medición y evaluación funcional constituyen una de las acciones fisioterapéuticas más importantes para mejorar las prácticas terapéuticas. Durante los últimos años ha habido un considerable incremento de los estudios de intervención, especialmente en aquellos pacientes portadores de lesiones cerebrales (Kappelle, 1998).

El desarrollo alcanzado por las neurociencias y la informática ha permitido que los métodos convencionales de medición y evaluación funcional sean sustituidos paulatinamente por programas computarizados especializados que proporcionan innumerables ventajas al proceso rehabilitador.

Los profesionales de las ciencias de la salud que tienen como interés mejorar la capacidad de movimiento se interesan por el fenómeno del desplazamiento corporal apoyándose en procesos de medición y de evaluación cinemática. Bajo este contexto es necesario indagar en el diseño de instrumentos de evaluación de movimiento que sean cada vez más objetivos y que permitan aplicar intervenciones cada vez más efectivas. En este sentido, se cuenta con un abanico de posibilidades entre las cuales están: el uso de grabaciones en video, la digitalización de imágenes por computador, la kinegramática como método de seguimiento del movimiento en imágenes cuadro a cuadro y su esquematización, el acelerómetro, la electrogoniometría, junto con la creación de programas "software", los cuales permiten realizar un análisis biomecánico detallado de la ejecución del movimiento corporal humano en un determinado contexto.

En Colombia, esta clase de análisis cinemático de movimiento se ha aplicado de manera incipien- 
te en áreas de la clínica y el deporte, donde se requiere de métodos evaluativos que faciliten la emisión de juicios confiables en cuanto a la descripción del movimiento.

Es aquí, donde la Fisioterapia, como disciplina de la salud aplicada, debe emplear herramientas encaminadas al mejoramiento de la comprensión del gesto de movimiento, permitiendo así su utilización en diferentes espacios, para identificar las alteraciones de movimiento, el correcto diseño de aditamentos y los métodos de habilitación apropiados para cada persona.

Para ello se plantea el objetivo general de esta investigación: realizar un análisis del APAS como instrumento de evaluación fisioterapéutica de la marcha en un paciente con Parálisis Cerebral mejorando los procesos de diagnóstico y de intervención dentro de la fase de secuelas de la enfermedad.

Los objetivos específicos fueron: conceptuar alrededor de los procesos de medición y evaluación utilizando instrumentos de la informática para el análisis de movimiento desde una perspectiva fisioterapéutica; proponer procesos de medición cada vez más objetivos que respalden los procedimientos y el diagnóstico fisioterapéutico aportando a la conformación de una unidad de criterio común en el análisis de la marcha; describir el comportamiento de las variables cinemáticas en los planos sagital, frontal y coronal, que presenta la marcha espástica en el paciente; identificar variables de medición y seguimiento del comportamiento cinemático que permitan aportar a la comprensión de la marcha, con el fin de establecer los aspectos que en este sentido pueden influir positiva o negativamente en el proceso de rehabilitación de los mismos; demostrar la importancia que tiene el uso de instrumentos especializados por parte de los fisioterapeutas a fin de mejorar los niveles de confiabilidad y validez de los procesos de medición y evaluación de la marcha como complemento de la valoración de la marcha tradicional; junto con el diagnóstico y pronóstico funcional mejorar la capacidad técnica del profesional en Fisioterapia.

Para ello se proponen las siguientes variables:

La cinemática: descripción del movimiento en la marcha en cuanto al desplazamiento angular de las articulaciones en los tres planos y la velocidad del desplazamiento articular de los miembros inferiores.

Determinantes de la marcha: aspectos específicos de la marcha como el Tilt de pelvis, la longitud del paso, la longitud de zancada, el ancho de paso y la cadencia en la marcha.

Tiempo de duración: en términos de duración en tiempo de cada ciclo de marcha de cada extremidad.

\section{Método}

\section{Tipo de investigación}

Este estudio fue descriptivo, estudio de caso.

\section{Participante}

Sujeto adulto de 32 años de edad, de sexo masculino, con secuelas de parálisis cerebral, quien presenta una marcha espástica sin ayudas externas.

\section{Instrumento}

Evaluación fisioterapéutica junto con una valoración funcional de su marcha con un previo consentimiento informado del paciente. Instrumento para la evaluación de la marcha: se empleó un software especializado (APAS: Ariel Performance Analysis System), el cual es un sistema computarizado para el análisis biomecánico y el estudio del movimiento humano, basado en la captura en video de imágenes de la marcha con múltiples cámaras simultáneamente. Dicho estudio se realizó en el Instituto CIREC (Centro Inte- 
gral de Rehabilitación de Colombia) dentro del laboratorio de análisis de movimiento.

Para la ejecución de la prueba previamente se diseñaron unos marcadores adhesivos con capacidad de difracción y de reflexión de la luz que puestos estratégicamente en puntos de cada segmento óseo del tren inferior es posible hacer un seguimiento osteocinemático del desplazamiento articular en el espacio. (Véase tabla 1).

\section{TABLA 1}

Seguimiento OSTEOCINEMÁtico

\begin{tabular}{lllll}
\hline $\mathbf{N}^{\circ}$ & Nombre & Cant. & Ubicación \\
\hline 1 & 13 & $\begin{array}{l}\text { Espina ilíaca antero } \\
\text { superior }\end{array}$ & 2 & $\begin{array}{l}\text { Miembros } \\
\text { inferiores }\end{array}$ \\
\hline 2 & 14 & Trocante mayor & 2 & $\begin{array}{l}\text { Miembros } \\
\text { inferiores }\end{array}$ \\
\hline 3 & 15 & Cóndilos externos & 2 & $\begin{array}{l}\text { Miembros } \\
\text { inferiores }\end{array}$ \\
\hline 4 & 16 & Rótula & 2 & $\begin{array}{l}\text { Miembros } \\
\text { inferiores }\end{array}$ \\
\hline 5 & 17 & $\begin{array}{l}\text { Maléolos externos } \\
\text { Miembros } \\
\text { inferiores }\end{array}$ \\
\hline 6 & 18 & $\begin{array}{l}\text { Talón (a la altura del } \\
\text { calcáneo bilateral) }\end{array}$ & 2 & $\begin{array}{l}\text { Miembros } \\
\text { inferiores }\end{array}$ \\
\hline 7 & 19 & $\begin{array}{l}\text { Articulación metatarso } \\
\text { falángica lateral del } \\
\text { quinto dedo }\end{array}$ & 2 & $\begin{array}{l}\text { Miembros } \\
\text { inferiores }\end{array}$ \\
\hline
\end{tabular}

Número total de marcadores 14

Una vez realizada la prueba, se seleccionaron las tomas a las que se aplicaría el análisis. El criterio de selección principal fue la naturalidad de ejecución del movimiento, por lo que se recomienda escoger la tercera toma, debido a que se espera que en ésta el sujeto se encuentre familiarizado con el nuevo ambiente. Aunque se recomienda la tercera toma, la escogencia es un factor que se basa principalmente en factores cualitativos. Otro aspecto que se debe tener en cuenta en la selección de la toma es el de la calidad de la ima- gen de cada video, debido a que en ocasiones aparecen haces de luz que impiden visualizar correctamente los marcadores.

Seleccionadas las tomas, se realizó el proceso de edición de videos, con lo que se buscó que los videos iniciaran y finalizaran al mismo tiempo. Este proceso consiste en dos pasos que se ejecutan en el módulo TRIMMER del software APAS. El primer paso es realizar la sincronización de los videos, en la cual se le indica al programa para cada una de las cinco cámaras, el cuadro en el que se presenta un evento común a todas las cámaras y que ocurre en un determinado tiempo. En este caso se trata del momento en que un conjunto de LEDS se encienden durante la filmación. Con esta referencia, el módulo TRIMMER es capaz de establecer una coordenada de tiempo común y coherente para los cinco videos. El siguiente paso es el de cortar dichos videos, para que queden de la misma longitud, iniciando en el momento en el que el sujeto hace contacto con el piso y finalizando en el momento que se separa de ésta, dentro del ciclo de la marcha (Quintero, 2002).

El paso siguiente fue la digitalización del movimiento, el cual consiste en indicarle, a manera de usuario, al módulo DIGITAZE de APAS, cuadro por cuadro, para cada una de las 5 cámaras, y la ubicación en pantalla de cada marcador. Este es el trabajo más dispendioso de todo el proceso, debido a que cada uno de los cinco videos de las dos tomas seleccionadas, posee en promedio una cantidad de 400 cuadros y en cada uno de estos cuadros aparece en promedio la mitad del conjunto de marcadores. Con esta información, APAS realiza triangulaciones de las posiciones de los marcadores, para así construir una representación tridimensional del movimiento de cada uno de éstos. El resultado es un archivo de texto en el cual se encuentran las coordenadas cartesianas en bruto y suavizadas con splines, con respecto al cero establecido y cuadro a cuadro para cada marcador. 
Finalmente, el archivo de texto fue leído por el programa de cálculo de variables, el cual transforma la información del archivo de texto en una matriz en Visual Basic, a partir de la cual se efectúan los cálculos de las variables del modelo cinemático.

El programa de cálculo de variables muestra sus resultados por medio de gráficos interactivos en los cuales se puede apreciar el valor de cada variable en determinado instante de tiempo, la fase de la marcha en la que se encuentra y realizar comparaciones con otras variables; también es posible exportar los datos a un formato de hoja de cálculo, si se necesita algún tipo de cálculo posterior.

\section{Resultados y análisis}

A continuación se presentan los resultados generados por la aplicación del programa elaborado en Visual Basic que realiza el cálculo de las variables cinemáticas, presenta un panel principal mostrado en la Figura 1, en el cual se tienen las opciones de cargar el archivo de los datos registrados del paciente, lo que permitirá observar el comportamiento de las variables cinemáticas, y exportar sus valores numéricos en un plano cartesiano, donde se describe el comportamiento de cada articulación del tren inferior en el tiempo del ciclo de la marcha patológica.

El valor agregado es que el sistema presenta datos cualitativos y cuantitativos que permiten estimar dentro de una escala nominal y categorial los datos presentados favoreciendo la interpretación y análisis. Aunque el uso de este sistema de análisis de datos especializado es costoso, el beneficio es la precisión para la evaluación y diagnóstico de las disfunciones motoras, junto con el análisis comparativo, que se realiza gracias a las interfaces y gráficas en el tiempo

La interfaz gráfica interactiva permite que se realice el estudio descriptivo de la cinemática y se observe el comportamiento de cada variable

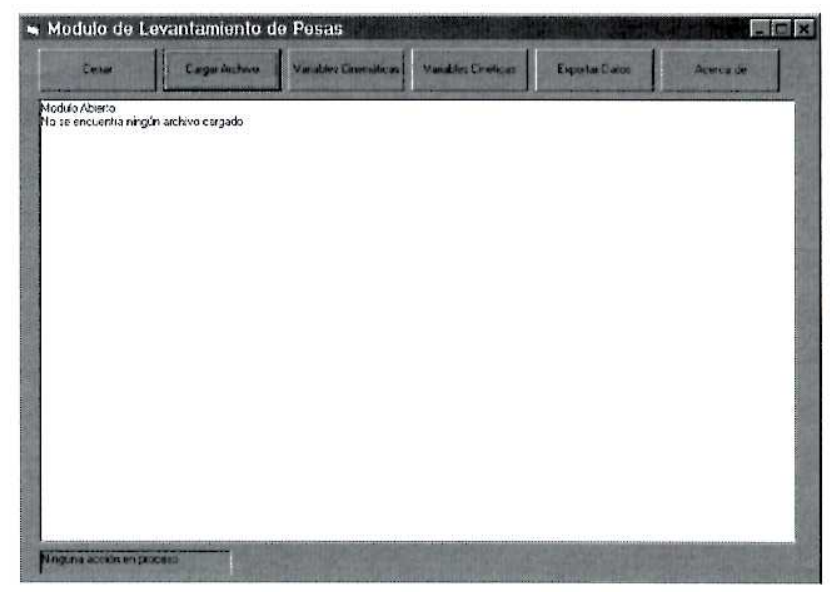

Figura 1. Interfaz principal del programa elaborado para el cálculo de las variables.

cinemática a lo largo del tiempo, indicando para un instante específico el valor numérico de dicha variable así como la fase del movimiento en la que se encuentra. Igualmente, para cada variable se puede comparar el proceder del hemicuerpo derecho con el izquierdo, ya que se muestran en cada gráfica estos dos resultados cuando es aplicable.

También permite comparar la conducta de una determinada variable con otras adicionales, permitiendo observar más de una gráfica al mismo tiempo. En la figura 2 se muestra un ejemplo de cómo se observa la interfaz de gráficos del programa.

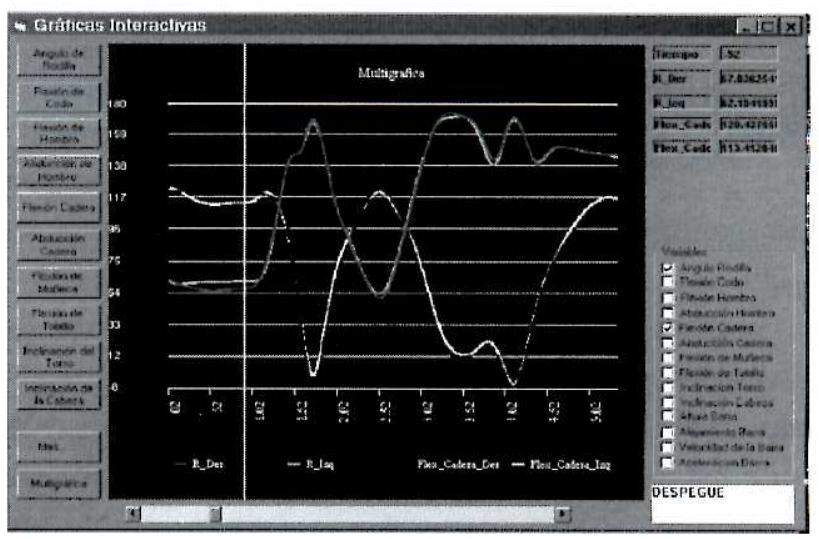

Figura 2. Interfaz de gráfico. 
El programa, permite exportar los datos a una hoja de cálculo, a partir de la cual se puede realizar un procesamiento adicional a los mismos. El comando de exportación permite seleccionar la variable específica de interés.

Para la presentación de los resultados de las variables cinemáticas de manera cuantitativa se presentarán primero los resultados de la evaluación fisioterapéutica de los aspectos de una valoración clínica que se tuvieron para el caso como marco de referencia, antes de presentar las variables relacionadas con la marcha.

\section{Valoración clínica tradicional}

Es un paciente con diagnóstico de parálisis cerebral espástica. Entre los antecedentes relevantes destaca la realización de una capsulotomía posterior y alargamiento de isquiotibiales para corregir una deformidad en flexión de la rodilla izquierda, este procedimiento se realizó hace 25 años, aproximadamente.

El examen neurológico de sus extremidades revela una espasticidad pura que afecta sus cuatro extremidades, con predominio de los miembros inferiores y, entre ellos, del miembro inferior derecho. El examen de control muscular selectivo muestra un severo compromiso global, especialmente en rodillas y pies. En las caderas el control selectivo tan sólo es aceptable para la flexión.

Las pruebas para evaluar equilibrio demuestran deficiencias en todos los sentidos; sin embargo, logra deambular sin ayudas externas ni uso de férulas.

Los hallazgos más llamativos son la pérdida de movilidad articular y la presencia de retracciones marcadas en todas las articulaciones principales, así como el hallazgo de deformidades en flexión de cadera y rodillas. En las articulaciones subastragalinas está limitada la eversión en el pie derecho, y hay deformidad leve en inversión en el pie izquierdo.

Hay compromiso importante de la fuerza, en especial en el miembro inferior izquierdo, en el que pie y tobillo no tienen musculatura funcional. El tobillo y pie derechos tienen calificación global de cuarta. La fuerza de rodillas y caderas está disminuida pero dentro de rangos funcionales para ambas extremidades.

Las deformidades en flexión de las extremidades impiden realizar una medición confiable de la longitud de los miembros inferiores, aunque no se encuentran diferencias clínicamente evidentes. El perfil torsional muestra un aumento importante de la anteversión femoral con una torsión tibial externa dentro de límites normales. La conformación angular es normal. Otro hallazgo llamativo es la presencia de rótulas altas asociadas a paresia del cuádriceps.

Las radiografías de caderas muestran que ambas articulaciones están bien reducidas y cubiertas, tienen aspecto valgo. Las rodillas están deformadas en flexión con rótulas altas y calcificación del tendón rotuliano.

\section{Fases de la marcha}

Para el análisis del ciclo de marcha éste debe ser expresado en una terminología técnica, para ello los resultados se presentan en porcentaje de distribución del total del tiempo que dura el ciclo de marcha.

El paciente con secuelas de parálisis cerebral con una alteración de la marcha busca la adaptación a factores compensatorios que hacen difícil realizar "una marcha normal", llevándolo a caminar con esfuerzo por períodos cortos de tiempo y con tiempos de descanso prolongados alterando su capacidad de desplazamiento. La asi- 
metría en cuanto a la velocidad del desplazamiento y la ejecución de cada fase de la marcha lleva a un elevado gasto energético para tratar de mantener la línea de gravedad dentro de la base de sustentación.

\section{Índice de consumo de energía}

El consumo de energía de la marcha aumentó significativamente debido a las compensaciones musculares y alteraciones posturales que generan un aumento metabólico para la ejecución de la actividad. (Véase tabla 2).

\section{TABLA 2}

TIEMPO DE DURACIÓN DEL PROTOCOLO

\begin{tabular}{|c|l|l|l|}
\hline \multicolumn{2}{|c|}{ Actividad } & Instrumento & Duración \\
\hline 1 & $\begin{array}{l}\text { Colocación } \\
\text { del set de } \\
\text { marcadores }\end{array}$ & 14 Marcadores & 15 min. \\
\hline 2 & $\begin{array}{l}\text { Alistamiento } \\
\text { de cámaras }\end{array}$ & $\begin{array}{l}\text { Cámaras, } \\
\text { lámparas, } \\
\text { soportes }\end{array}$ & 15 min. \\
\hline 3 & $\begin{array}{l}\text { Indicaciones de } \\
\text { desplazamiento } \\
\text { sobre la } \\
\text { plataforma de } \\
\text { marcha }\end{array}$ & 10 min. \\
\hline 4 & $\begin{array}{l}\text { Calibración } \\
\text { Cubo de } \\
\text { calibración }\end{array}$ & 2 min. \\
\hline 5 & $\begin{array}{l}\text { Filmación } \\
\text { (3 tomas) }\end{array}$ & $\begin{array}{l}5 \text { cámaras de } \\
\text { video digital }\end{array}$ & 10 min. \\
\hline \multicolumn{3}{|l|}{$\begin{array}{l}\text { Tiempo estimado del proceso } \\
\text { de filmación }\end{array}$} & 52 min. \\
\hline
\end{tabular}

Este gasto de energía estimado a través de la correlación en término de frecuencia cardiaca sobre distancia por tiempo, demuestra que el aumento en el trabajo de la bomba cardiaca fue de casi el $100 \%$ en el consumo de energía por parte de los sistemas corporales durante la actividad.

\section{Variables cinemáticas}

Para la descripción y el comportamiento de las variables cinemáticas del tren inferior durante el desarrollo del ciclo de la marcha en el paciente los resultados se presentan en términos de gráficos describiéndose el comportamiento articular por su distribución por planos sagital, coronal y transverso mencionando el comportamiento más relevante de cada articulación de los miembros inferiores.

\section{Cinemática en el plano sagital}

La pelvis está permanentemente basculada hacia adelante en una anteversión de 15 grados, acompañada con un aumento en la oscilación de ésta. Esto se explica por la presencia de contractura muscular del músculo psoas iliaco generando una deformidad en flexión lo que pone en desventaja mecánica y por la necesidad de reclutar la musculatura paravertebral del tronco para la propulsión.

La cadera izquierda mantiene un aumento en la flexión durante el contacto inicial en el choque de talón, con imposibilidad para la extensión completa sobre todo en la fase de apoyo terminal en la cadera derecha. Esto es bilateral, pero más marcado en el miembro inferior izquierdo. La cadera izquierda presenta una tendencia a la aducción sobre todo en la fase de apoyo, mientras que la cadera contraria se mantiene en ligera abducción. Con relación a la rotación de la articulación coxofemoral se observa que la articulación izquierda mantiene externa durante todo el ciclo, mientras la derecha mantiene una rotación externa sobre todo en la fase de apoyo. (Véase tabla 3).

En cuanto a la rodilla, las curvas en la gráfica en la fase de balanceo se encuentran asimétricas por la posición contraria que aumenta en la fase de balanceo, en la fase de apoyo se encuentra la rodilla derecha en una flexión mantenida debido a contractura articular. Esto refleja una deformi- 
dad articular asociada a retracción de isquiotibiales, paresia del cuádriceps y coespasticidad entre recto anterior e isquiotibiales.

TABLA 3

Tiempo de duración del PROCESO de DIGITALIZACIÓN

\begin{tabular}{|c|l|l|l|}
\hline $\begin{array}{c}\text { Actividad } \\
\text { Número Nombre }\end{array}$ & Instrumento & Duración \\
\hline 1 & $\begin{array}{l}\text { Selección de } \\
\text { las tomas }\end{array}$ & Cualitativo & $15 \mathrm{~min}$. \\
\hline 2 & $\begin{array}{l}\text { Edición de } \\
\text { videos }\end{array}$ & $\begin{array}{l}\text { APAS } \\
\text { TRIMMER }\end{array}$ & $30 \mathrm{~min}$. \\
\hline 3 & $\begin{array}{l}\text { Digitalización } \\
\text { del movimiento }\end{array}$ & $\begin{array}{l}\text { APAS } \\
\text { DIGITAZE }\end{array}$ & 7 días \\
\hline 4 & $\begin{array}{l}\text { Cálculo de } \\
\text { variables } \\
\text { cinemáticas }\end{array}$ & $\begin{array}{l}\text { Programa } \\
\text { para análisis } \\
\text { cinemático }\end{array}$ & 10 min. \\
\hline $\begin{array}{l}\text { Tiempo estimado del procesamiento de video y datos } \\
8 \text { días }\end{array}$ \\
\hline \multicolumn{2}{|c|}{} \\
\hline
\end{tabular}

En referencia al tobillo, en ambos miembros inferiores el contacto inicial es con la punta, igualmente en ambas extremidades se observa un despegue precoz del talón por espasticidad de los gastronemius. En el tobillo izquierdo se encuentra pie equino permanente mientras que en el tobillo derecho logra la posición plantígrada e incluso tiene comportamiento talo en el apoyo medio. (Véase gráfica 4).

\section{TABLA 4}

DistribuCIÓN PORCENTUAL DEL CIClo DE MARCHA

\begin{tabular}{lccc}
\hline $\begin{array}{c}\text { Fases de } \\
\text { la }\end{array}$ & $\begin{array}{c}\text { Rango de } \\
\text { normalidad } \\
\text { marcha }\end{array}$ & $\begin{array}{c}\text { Extremidad } \\
\text { derecha } \\
\text { del paciente } \\
\text { \% }\end{array}$ & $\begin{array}{c}\text { Extremidad } \\
\text { izquierda }\end{array}$ \\
\hline Descarga & 20 & 30.3 & $\%$ \\
$\begin{array}{l}\text { Apoyo completo } \\
\text { Balanceo }\end{array}$ & 40 & 40.9 & 30.2 \\
\hline
\end{tabular}

\section{Cinemática en el plano coronal}

Pelvis y cadera: estas curvas demuestran una pobre participación de los abductores bilateral más evidente en el lado izquierdo durante todo el ciclo de la marcha. (Véase gráfica 5)

\section{TABla 5}

Gasto energético durante el Ciclo de Marcha

\begin{tabular}{cc}
\hline $\begin{array}{c}\text { Gasto energético } \\
\text { normal }\end{array}$ & $\begin{array}{c}\text { Gasto energético } \\
\text { del paciente }\end{array}$ \\
El ideal es 0.4 latidos & 0.791 latidos \\
por $\mathrm{min} / \mathrm{mts} \mathrm{sg}$ & por $\mathrm{min} / \mathrm{mts} \mathrm{sg}$ \\
\hline
\end{tabular}

Rodilla: esta articulación no se observa en el plano, por tanto, no se describe su comportamiento.

Tobillo: las articulaciones subastragalinas llegan a posición neutra durante la fase de apoyo completo y durante el balance discurren en discreto varo. Esto demuestra que posteriormente es importante fortalecer el tibial posterior para evitar el riesgo de que se desarrolle un pie plano valgo si se decide realizar un procedimiento quirúrgico.

\section{Cinemática en el plano transverso}

Pelvis: las curvas son marcadamente asimétricas con rezago de la hemipelvis izquierda, esto se explica porque la anteversión femoral izquierda es mayor lo que obliga a un movimiento oscilatorio durante el ciclo de la marcha.

Rodilla: en las curvas se muestra una progresión marcadamente negativa de ambas rótulas, esto es más evidente durante el final del balanceo por contractura muscular del gracilis. Este fenómeno obliga a utilizar el mecanismo de circunducción que será descrito posteriormente. (Véase gráfica 6). 
TABLA 6

Parámetros complementarios de la marcha

\begin{tabular}{lcc}
\hline $\begin{array}{l}\text { Parámetros de } \\
\text { la marcha }\end{array}$ & *Valor normal & $\begin{array}{c}\text { Valores en el } \\
\text { paciente }\end{array}$ \\
\hline Duración de zancada & $0.8 \mathrm{sg}$ & $1.26 \mathrm{sg}$ \\
Longitud de zancada & $120 \mathrm{~cm}$ & $103 \mathrm{~cm}$ \\
Duración de paso & $0.4 \mathrm{sg}$ & $0.6 \mathrm{sg}$ \\
Longitud de paso & $55 \mathrm{~cm}$ & $49.9 \mathrm{~cm}$ \\
Velocidad de la marcha & $50 \mathrm{~cm} * \mathrm{sg}$ & $78.7 \mathrm{~cm} * \mathrm{sg}$ \\
\hline
\end{tabular}

*Valores tomados para un ciclo de marcha normal de un adulto de $70 \mathrm{~kg}$.

Tobillo: la progresión de los tobillos con respecto a las rótulas es normal, esto sugiere que el perfil torsional de la tibia es normal.

En general teniendo en cuenta los tres planos de movimiento en el espacio demuestra una importante paresia global, especialmente en el miembro inferior izquierdo. La propulsión de la marcha del sujeto depende casi exclusivamente de los extensores y flexores de la cadera derecha. Esta situación está agravada por la presencia de momentos musculares anormales, extensores en la rodilla y flexores en la cadera, que son consecuencia de las deformidades articulares y que aumentan el consumo de energía.

\section{Características identificadas de la marcha}

Frente al análisis de las gráficas se puede identificar algunos patrones de movimiento que son característicos de la marcha espástica como:

\section{Inestabilidad de la rodilla}

Se encuentra una asimetría en las articulaciones de la rodilla durante el choque de talón en la fase de apoyo medio, en los siguientes aspectos:
- Inclinación lateral del tronco:

El tronco se inclina hacia el lado contrario de la extremidad que se encuentra en apoyo. Este movimiento oscilatorio del tronco desvía el centro de gravedad lateralmente del individuo hacia el lado izquierdo sobre todo durante el contacto inicial hasta el apoyo completo. La inclinación lateral del tronco por lo general, se presenta cuando el sujeto camina en circunducción. Los mismos factores que producen la marcha en abducción pueden ser los responsables de esta inclinación lateral del tronco.

- Movimiento de rotación en el período de balanceo:

Al despegar el pie, el talón se mueve medialmente en el momento de despegar el pie del suelo, causado por un varo del pie generado probablemente por un pie equino varo compensado con una rotación externa del fémur.

- Desplazamiento vertical del cuerpo:

El cuerpo desciende tan pronto el peso es transferido a la pierna durante el período de balanceo de la pierna que se encuentra en el aire, al transferir el peso y por consiguiente el apoyo a la extremidad contraria se genera un desplazamiento brusco descendiente vertical del cuerpo.

\section{Lordosis lumbar}

Se evidencia un aumento de la convexidad lumbar durante el período de apoyo de las extremidades inferiores debido a la espasticidad del psoas iliaco, así:

- Circunducción o abducción de los miembros inferiores:

La extremidad sigue un desplazamiento curvo en sentido externo lateral durante el período de balanceo en ambas extremidades relacionadas con la prótesis por fricción excesiva de la articulación de la rodilla que obliga 
al sujeto a oscilarla hacia fuera para no rozar el suelo.

- Parámetros complementarios de la marcha:

Los parámetros complementarios de la marcha hacen referencia a las variables de tiempo y desplazamiento, donde se evidencia la disminución en la velocidad del desplazamiento del sujeto evaluado comparado con valores normales y la disminución en las distancias recorridas en términos de paso y zancadas, lo que refuerza la idea del aumento en el gasto de energía.

- Resultados de la evaluación general con el apoyo del APAS:

Pies con equino varo dinámico. El pie derecho con comportamiento talo y equino; junto con hipoactividad del tibial posterior está defendiendo del colapso del pie durante el apoyo lo que genera que el apoyo se dé con el antepié. Deformidad en flexión bilateral de rodillas por imbalance muscular de los isquiotibiales y cuádriceps por la hipertonía marcada y las contracturas articulares, lo que limita el rango articular. Retracción del recto interno y aductores bilateralmente, lo que genera un patrón de tijera moderada durante la marcha. Deformidad mantenida en flexión de las caderas por contractura muscular de psoas iliaco.

\section{Discusión}

La parálisis cerebral se considera actualmente como la enfermedad neurológica más grave de la infancia durante el siglo XXI, la cual tiene incidencia y prevalencia cada vez mayor en la población colombiana. Este estudio descriptivo, pone en evidencia la importancia de reflexionar alrededor de las necesidades y procedimientos requeridos por los pacientes que se encuentran en fase crónica de la enfermedad, buscando alternativas de evaluación que permitan diagnosticar con mayor precisión las deficiencias motoras y las compensaciones del aparato osteomuscular.

Como se menciona en los objetivos específicos al contrastar las aproximaciones teóricas y conceptuales iniciales con el ejercicio en el análisis de caso, se puede reconocer la importancia que toman los avances tecnológicos en rehabilitación, particularmente en los procesos de medición y evaluación como punto de partida en la intervención del grupo interdisciplinario, específicamente desde la fisioterapia.

En esta medida los recursos tecnológicos utilizados en los procesos mencionados, permiten un mayor nivel de confiabilidad y validez en el registro e interpretación de datos que permiten objetivizar el diagnóstico y la posterior intervención fisioterapéutica. Asimismo, al complementar esta tecnología con la medición y evaluación tradicional de la marcha se generará un lenguaje técnico común que aporta al desarrollo profesional y su consolidación dentro de la comunidad científica.

Por tanto, al describir el desplazamiento angular de las articulaciones de los miembros inferiores del sujeto durante el ciclo de la marcha, éstos se compararon con datos generales de una marcha normal basándose en la bibliografía presentada, lo que permite demostrar la precisión que tiene el APAS como instrumento de apoyo en la valoración fisioterapéutica.

Al describir el comportamiento de las variables cinemáticas en el espacio durante el recorrido de la marcha se puede decir que el APAS es un instrumento de rigor que sí apoya los procesos de medición utilizados de manera tradicional y la identificación de las deficiencias motoras y las compensaciones generadas por las lesiones neurológicas. Con el tiempo este instrumento se utilizará en mayor medida en la descripción de las deficiencias estructurales, funcionales y de restricción motora en el diagnóstico fisioterapéu- 
tico y sus implicaciones cinéticas para la instauración de la discapacidad física en términos de pronóstico funcional.

El movimiento humano requiere de un estudio detallado, donde se muestre todos los componentes básicos incluyendo la aplicación de la biomecánica. La cinemática de la marcha se puede llevar a cabo desde un enfoque cuantitativo o cualitativo. El análisis cuantitativo involucra la descripción de un movimiento o de sus partes en términos numéricos, y es medido por medio de instrumentación (software). El análisis cualitativo tiene en cuenta la calidad del movimiento mediante la valoración observacional de la marcha, llevando a cabo una descripción visual del comportamiento de las extremidades inferiores de un individuo, la movilidad del tronco y la pelvis durante la ambulación.

La marcha se analiza de forma tridimensional debido a que los movimientos se realizan en diferentes planos y ejes, por tal razón requiere ser analizada en las dimensiones: coronal, sagital y transversal.

El plano coronal también se denomina plano frontal y divide el cuerpo en una porción anterior y una posterior, su nombre se deriva de la sutura coronal del cráneo. Este plano se une al eje sagital para permitir los movimientos de abducción y aducción. Este plano, permite evaluar la escoliosis y la discrepancia de longitud de los miembros inferiores, la marcha de trendlemburg, el valgo y el varo de rodilla, entre otros.

El plano sagital también se denomina plano anteroposterior, es el que divide el cuerpo en las mitades derecha e izquierda, se une al eje transversal para permitir los movimientos de flexión y extensión.

Este plano permite observar el grado de lordosis lumbar, y contracturas en flexión de cadera y rodilla. A nivel del tobillo permite mirar cómo el paciente realiza el contacto inicial. Tiene una influencia decisiva en la longitud del paso y por tanto, en la eficacia de la marcha.

El plano transversal u horizontal divide el cuerpo en dos porciones superior e inferior. Se une con el eje vertical permitiendo los movimientos de rotación. Los datos de este plano muestran el ángulo de progresión. La exploración de la marcha en este plano permite observar deformidades torsionales en fémur y tibia.

Por lo tanto, el punto de intersección de los tres planos medios del cuerpo se denomina centro de gravedad en la marcha normal del ser humano, la trayectoria que sigue el centro de gravedad corporal "traza" una curva uniforme regular que se desplaza hacia arriba y abajo en el plano frontal, con una distancia promedio de ascenso y descenso de $5 \mathrm{~cm}$. El punto bajo se alcanza en la fase de doble apoyo en que ambos pies están en contacto con el suelo y el punto alto en la fase de postura media.

El centro de gravedad corporal también se desplaza hacia los lados en el plano horizontal durante la locomoción, y la distancia laterolateral total en ese sentido es de $5 \mathrm{~cm}$. El movimiento se hace hacia el miembro que soporta peso y alcanza su límite lateral en la postura media. Cuando se combina los movimientos horizontal y vertical del centro de gravedad describe una curva sinusoidal doble.

De esta forma, el centro de gravedad es un factor a tener en cuenta en todo el proceso del análisis de la marcha, ya que su alteración implica un cambio en el movimiento del individuo y un aumento del gasto energético durante la locomoción.

Además, no sólo se debe limitar a nombrar el tipo de desviación de marcha sino la extensión y la calidad, para esto se debe conocer las características normales de la locomoción humana que será el parámetro a tener en cuenta para hacer la medición, la kinesiología, biomecánica y las ca- 
racterísticas funcionales y estructurales de los componentes protésicos.

Sin embargo, el paciente con PC con una desviación de la marcha busca la adaptación a factores compensatorios que hacen difícil realizar "una marcha normal", llevándolo a caminar con esfuerzo por períodos cortos de tiempo, pero a expensas de un elevado gasto energético.

\section{Conclusiones}

Este estudio demostró las ventajas del uso del APAS como apoyo fisioterapeuta para la valoración de la marcha funcional.

En este estudio de caso se demostró que el período postural del ciclo de marcha se encuentra aumentado, lo que significa que el sujeto no realiza un total traslado de peso en la extremidad derecha, generando una alteración en las fases y las tareas funcionales de la marcha.

Se propone practicar un estudio que incluya una muestra significativa de sujetos con disfunciones motoras de la marcha para estudiar las similitudes y diferencias que tiene el patrón de marcha en este tipo de población y al mismo tiempo establecer las variaciones que sean necesarias en el software para un análisis más detallado.

El APAS permite de manera precisa el seguimiento de los movimientos de la pelvis que regula el movimiento del centro de gravedad en sentido horizontal, para este estudio de caso se evidenció movimientos paradójicos lo que significa control inadecuado del centro de gravedad y esto se ve reflejado en un aumento del consumo de energía sobre el ideal, lo que demuestra la importancia del manejo fisioterapéutico de los movimientos de la pelvis en el proceso de rehabilitación.

El APAS proporciona una evaluación del desplazamiento angular de los miembros inferiores en los tres planos espaciales (plano sagital, frontal y transverso), lo que da una visión tridimensional del movimiento corporal humano, detectando alteraciones anatómicas y funcionales que no pueden ser determinadas en una evaluación de la marcha tradicional por observación del clínico corroborando lo propuesto por Howell V., (1983).

El miembro inferior derecho altera su desempeño biomecánico en forma compensatoria debido a la presencia de espasticidad del miembro lo que afecta el ciclo de marcha.

Este análisis con base en las gráficas del APAS permite revisar la marcha del sujeto tantas veces como sea necesario sin que esté presente, además de observar detalladamente las desviaciones de la marcha y comparar la evolución de la persona durante todas las fases de la rehabilitación.

Utilizar esta tecnología en la intervención fisioterapéutica con personas con secuelas de PC, aporta datos exactos en todas las variables descritas para este estudio, lo que puede mejorar el abordaje de estas personas en fases posteriores a una corrección quirúrgica, proporcionando aspectos a trabajar en la reeducación del patrón de marcha

Este estudio es un punto de partida para futuras investigaciones, ya que hasta el momento no hay evidencia escrita desde Fisioterapia de este tipo de estudio en Colombia.

Esta investigación brinda la posibilidad de interactuar con otros profesionales diferentes del área de la salud, además, de requerir dedicación, compromiso y profundización de los conceptos necesarios para entender el movimiento corporal humano desde la cinemática y su expresión más compleja como es la "marcha humana".

\section{Referencias}

Adams, H.P.,; KaPelle, L.J.; BILleR, J. 1995. Ischemic in Young adults. Arch Neurol; 52 (5); 491-5. 
Andersson C.; Mattsson E. 2001. Adults with cerebral palsy: a survey describing problems, needs, and resources. Dev Med Child Neurol; 43: 76-82.

Alcántara, P.; SÁnchez, M. 2000. Estimación del riesgo para natal de la parálisis cerebral. Revista Digital, Buenos Aires A.8, № 51 , agosto 2002.

Bagilet, D.; Luján, S.; Diab, M.; Biancardi, H. 2003. Factores de riesgo en pacientes con parálisis cerebral. www.sati.org.ar/revista/ 14/v14na-1htm

BARRINAGA, F. 1996. Cerebral infaction in people under 4 years. Cerebrovascular Disease; 6: 75-9.

BAYONA, H.; ToRo, J. 2001. Enfermedad de parálisis cerebral. Guías de manejo Basadas en la evidencia. Fundación Santa Fe de Bogotá.

Bradom, R. 1997. Physical medice and rehabilitation. Philadelphia: Sounders Company. $2^{\mathrm{a}}$ ed. 1996. p. 33, 83. ed. Ayyappa. Normal Human Locomotion. part I: basic concept and terminology. En: Journal of Prosthetics and Orthotics. EE.UU. vol. 9, No. 1, 10-2.

Bоватн, B. 1986. Actividad refleja anormal. Editorial Panamericana, 12-21.

Buades J. 2002. Human color segmentation and matching using real image sequences biomechanic model. Computer graphics and vision group. Department of Computer Science.

Cantani, F.; Benedetti M.G. 2003. Kinematic assessment of foot ankle complex. Biomechanics laboratory, Istituti Ortopedici Rizzoli, Roma, Italy.

Correa, J. 2002. Bases de la medición y evaluación fisioterapéutica desde una perspectiva positivista. Revista colombiana de rehabilitación, vol. 1, No. 1, 13-6, Bogotá, Colombia.

Duarte, J.; Andrade, M. 1998. Perspectiva del movimiento en la temática del análisis del gesto danzal. Monografía de la Universidad de Aragón, España, 2-3.
GAGE, J. 1991. Gait analysis in cerebral palsy. England: Mac Keith Press. $1^{\mathrm{a}}$ ed., 73.

German A., Romero, D. 2001. Análisis de la marcha mediante el protocolo ELICLINC. Universitat Ramon Llull.

GowtTzKe, B. 1999. Medicina deportiva, el cuerpo y sus movimientos, bases científicas. España, Barcelona: Paidotribo, $3^{\mathrm{a}}$ ed., 312-5.

HaRT, R.G.; Mזler, V. 1993. Cerebral infarction in young adult: A practical approach. Stroke, 14: $110-4$.

Hessler, H. 1996. Concepto de rehabilitación. En: Revista Iberoamericana de Rehabilitación y Medicina, vol. 46, 5-6.

Hoyos, S. 2000. Pautas del recién nacido. Editorial panamericana, 12-25.

Howell, V. 1993. Análisis computarizado del movimiento. Una introducción. En: Kinesiología. Chile, No. 37, 80-5.

Hurtado, J. 1998. Metodología de la investigación holística. Fundación Supla, 2a ed., 213.

KAPPELle, L. 1998. Prognosis of young adults with paralysis cerebral dis, 1996; 6: 75-9.

KRUSEN. 1993. Medicina física y rehabilitación. Médica Panamericana, España, $4^{\mathrm{a}}$ ed., 23, 28, 113 a la 117,1064 a la $1066,1078,1079,1089$, 1093, 1096,1097, 1100.

Lebouch, J. 1992. Hacia una conciencia del movimiento corporal humano. Paidós, Barcelona, 65.

LEVAu, B. 1991. Biomecánica del movimiento humano. Trillas, México, $1^{\mathrm{a}}$ ed. 15, 177, 180.

Lissner, Y.; WILliams, P. 1991. Biomecánica del movimiento humano. Trillas, México, 15.

Malagón, V. 1994. Tratado de ortopedia y fracturas. Celsus, Colombia, 18, 127, 1927, 1930, 1931.

Mena B. 1998. Control postural y habilidad motriz: una reflexión desde los puntos de vista neurofisiológico y biomecánico. Ascofi, Me- 
morias XVII Congreso Nacional de Fisioterapia, 12.

Miralles A. 1996. Biomecánica clínica del aparato locomotor, Editorial Salvat, España, 3-4.

Ministerio de Salud. 1998. Guía de manejo y atención de la parálisis cerebral. Imprenta Nacional.

Moreno-Gea, P.; Blanco-Sánchez, C. 2000. Hacia una teoría comprensiva de rehabilitación de funciones cerebrales como base de los programas de rehabilitación en enfermos con daño cerebral. Rev Neurol; 30: 779-83.

PARDo, G. 1997. Investigación en salud. Colombia: MCGraw-Hill, 123.

Perry, J. 1992. Gait analysis normal and pathologic function. EE.UU. Slack Incorporated, 362-5.

Pezzoti, E., Bniancolini, M. 2002. Modelización cad3 del cuerpo humano y aplicación para el análisis del movimiento. Congreso de Ingeniería. España.

Polit, D. 2000. Investigación científica en ciencias de la salud, principios y métodos. México: MacGraw-Hill, $6^{\text {a }}$ ed., 241.

PRICE, R. 1986. Las teorías del movimiento. Ed. Interamericana, España, 213-24.

Quintero, H. 2002. El diseño de un software para el análisis de la marcha. Tesis de maestría, Bogota, Colombia, 50-3.
Restrepo-Arbeláez. 1995. Rehabilitación en salud. Universidad de Antioquia, Colombia.

Robirn, S. 1995. Patología estructural y funcional. MacGraw-Hill Interamericana. España, $5^{\text {a }}$ ed., 746.

Rodríguez, A.R. 2000. Rehabilitación en niños con $P C$. Servicio de Rehabilitación y Medicina Física. Hospital Montecelo, Pontevedra.

Shigeru, U.; TAкehiko, Y. 1996. Reliability of computer based video motion analysis and electromyography analysis of sit to stand movement. Department of Physical Therapy, Gunma University School of Health Sciences. Journal Physical Therapy, 49-54.

SMITH, J. 1994. Biomechanics and physical therapy. EE.UU., 12.

Triana, A.; Restrepo, H. 1998. Actividad motora, posibilidad de cuerpo ser en el mundo. Memorias XVII Congreso Nacional de Fisioterapia. Ascofi, 17-9.

VIEL, E. 1999. Diagnóstico y pronóstico fisioterapéutico. Barcelona, España: Masson. S.A., 6.

WhitTLe, M. 1996. Gait analysis an introduction. USA: Planta. Tree, $2^{\mathrm{a}}$ ed., 177-8. 